\title{
Birds of a Feather Flock Together: But do Humans and Robots? A Meta-Analysis of Human and Robot Personality Matching
}

\author{
Connor Esterwood ${ }^{1}$, Kyle Essenmacher ${ }^{2}$, Han Yang ${ }^{2}$, Fanpan Zeng ${ }^{2}$, and Lionel P. Robert ${ }^{3}$
}

\begin{abstract}
Collaborative work between humans and robots holds great potential but, such potential is diminished should humans fail to accept robots as collaborators. One solution is to design robots to have a similar personality to their human collaborators. Typically, this is done by matching the human's and robot's personality using one or more of the Big Five Personality (BFI) traits. The results of this matching, however, have been mixed. This makes it difficult to know whether personality similarity promotes robot acceptance. To address this shortcoming, we conducted a systematic quantitative metaanalysis of 13 studies. Overall, the results support the assertion that matching personalities between humans and robots promotes robot acceptance.
\end{abstract}

\section{INTRODUCTION}

Humans and robots are expected to work collaboratively, yet there are doubts about whether humans will fully accept robots [1], [2], [3]. Human and robot collaboration is occurring across assembly lines, order fulfillment centers, and product inspections service centers [4]. For example, Amazon is expanding its workforce to include 15,000 robots a year in its fulfillment centers [4]. Despite this, there are growing concerns over whether humans will fully accept robots as collaborators [5], [6], [7], [8].

One solution to promoting robot acceptance is to design robots to have a similar personality to that of their human collaborators. Homophily, the tendency for people to be attracted to those who are similar to themselves, is used to explain why humans prefer interacting with other humans with a similar personality [9]. However, when applied in the context of HRI the results have been mixed. On one hand, personality similarities have led to positive humanrobot interactions [10], [11]; on the other hand dissimilarities in personalities have also led to positive interactions [12].

Currently, there is a lack of a systematic analysis of the impact of personality similarity in HRI. This makes it difficult to know whether similarity promotes robot acceptance. To address this shortcoming, we conducted a systematic quantitative meta-analysis consisting of 13 studies to answer the question: Does personality similarity between humans and robots promote robot acceptance? A meta-analysis allows us to answer this question in the face of conflicting or mixed

\footnotetext{
${ }^{1} \mathrm{PhD}$ student at the School of Information. The University of Michigan, 105 S State St, Ann Arbor, MI 48109, United States of America cte@umich.edu

${ }^{2}$ Student at the School of Information. The University of Michigan, 105 S State St, Ann Arbor, MI 48109, United States of America

3 Associate professor at the School of Information and a core faculty member at the Michigan Robotics institute. The University of Michigan, 105 S State St, Ann Arbor, MI 48109, United States of America lproberteumich.edu
}

results by leveraging multiple studies [2], [13]. In addition, a meta-analysis can help to identify gaps in the literature that need further study. Therefore, the goal of this paper was to provide insights into not only what has been done but also what should be done to advance this area.

\section{BACKGROUND}

\section{A. Big Five Personality Traits}

Personality describes traits that represent an individual's predisposition toward a behavior or object. Personality has been used to predict human attitudes, emotions and behaviors in many settings [14], [15], [16]. The Big Five personality traits are the most popular traits used in the study of HRI [14], [17]. The Big Five include: (1) extroversion which represents being sociable, gregarious and ambitious; (2) agreeableness which reflects attributes of kindness, consideration, likeability, and cooperation; (3) conscientiousness, which is characterized by self-control and a need for achievement and order; (4) emotional stability, which reflects being well adjusted, emotionally stable and secure; and (5) openness to experience, which is characterized by flexibility of thought and tolerance of new ideas [18], [19], [20].

\section{B. Personality Similarity in HRI}

Personality is an influential factor when considering human-robot interaction [21], [22], [23], [24]. More specifically, personality similarity between humans and robots based on one or more of the Big Five traits has been used to better understand HRI [14], [25]. The literature on humanrobot personality can be categorized into three types of findings. One, studies have found that similarity can lead to positive interactions. More specifically, humans prefer robots with matching personality traits over robots with different personality traits [10], [11]. Two, some studies found that dissimilarity can lead to positive interactions. These studies assumed that opposites attract [26], [12]. Three, context has also been presented as a key factor for determining whether similarity or dissimilarity leads to positive interactions. For example, one study found that task type helped to determine when humans preferred robots with similar personalities [27]. In sum, the literature has not found consistent results.

\section{METHOD}

\section{A. Search Process}

The literature search employed multiple searches via Google Scholar, the ACM Digital Library, IEEE Explore, and Scopus. 


\section{B. Search Terms}

Using nine search terms, we reviewed results on a pageby-page basis. This took the form of paging through search results and manually checking these results against a broad set of inclusion criteria until no single result on the list met the specified criteria. All results prior to the page with no relevant results were included in our initial screening. The specific search terms utilized were:

- (human) AND (robot) AND ("personality")

- ("human robot interaction") AND ("personality")

- ("HRI") AND ("personality")

- (human) AND (robot) AND (personality)

- ("human robot interaction") AND (personality)

- ("HRI") AND (personality)

- (robot and personality)

- (human robot interaction and personality)

- (HRI and personality)

Each page contained 10-25 results (depending on the database) by default. In total, we found 1,819 results across all of our searches before accounting for duplicate entries.

\section{De-duplication}

Search results were exported from Google Scholar in .bib format using "publish or perish" application [28] and imported into $\mathrm{R}$ for processing. The other databases' results were exported using their respective built-in tools. We conducted de-duplication using the revtools package [29]. Then, we removed duplicate articles on the basis of title using fuzzy matching and followed up with manual screening. After removing duplicates, we had 1,069 unique entries.

\section{Criteria for Study Inclusion}

We used a three-stage approach, starting with broad eligibility criteria and then applying progressively stricter criteria on the previously refined results. The initial eligibility criteria were used in the page-by-page review of search results and in all subsequent screenings. The secondary eligibility criteria were implemented in title screening, abstract screening, and full-text screening. The tertiary eligibility criteria were implemented in full-text screening. The exclusion criteria were used throughout all steps of this review.

Papers were initially selected for inclusion if they met three specific criteria. First, studies were required to be classified as articles or academic works. Second, studies were required to be written in the English language. The reason for excluding non-English-language publications relates to the lack of a specialist or translator on the review team. The third criterion for our initial eligibility was that the titles or abstracts retrieved were required to explicitly mention both the term "robot" and "personality."

At the secondary level, we selected papers on the basis of four additional eligibility criteria. First, studies were required to be empirical in nature and design. Second, these studies were required to use embodied physical action (EPA) robots. Third, studies were required to include measures of human or robot personality. Fourth, studies were eligible only if they involved humans interacting with an EPA robot.
At the third level we used criteria specific to the purposes of this paper. These eligibility criteria were that studies must examine human personality similarity/dissimilarity in some capacity and investigate their impact on outcomes broadly defined as acceptance. A fourth level of filtering took place for meta-analytic purposes and involved filtering out studies that did not report data that were useful for conducting a meta-analysis (effect sizes, sample size, etc.).

We excluded studies if they (1) focused on embodied virtual action (EVA; i.e. virtual agents), (2) focused on telepresence robots, (3) focused only on manipulating robot personality without examining its impact on humans, or (4) focused only on negative attitudes toward robots (NARS) as the personality trait of interest. The exclusion of studies that used the NARS scale was based on its use as a control variable in many studies (see [30], [25]).

\section{E. Screening Procedure}

Title screening was conducted manually in the revtools environment on the 1,069 unique entries previously identified. Screening was done only on the article title with author names and publication name hidden. Title screening was conducted based on the third criterion of the initial eligibility screening. Specifically, studies were included or excluded on the basis of the explicitly containing the terms "robot" and "personality." This screening identified 197 eligible studies. We conducted abstract screening manually in the revtools environment on the 197 eligible studies. Abstracts were extracted from Google Scholar and manually added to the data-set utilized by revtools. This screening incorporated all previous eligibility criteria in addition to the secondary eligibility criteria and led to the identification of 84 relevant studies.

In addition, we identified 50 other potential references from previously published review papers on the topic (see [25]). All papers identified via this means were reviewed in the same way as the papers identified by our search (title and abstract screening) and with identical criteria. Ultimately, 34 of the additional 50 references were found to be eligible for full-text screening.

Finally, each of the 118 selected papers were read in detail to determine their suitability based on all previously listed criteria and our tertiary eligibility criteria. After screening, 18 papers met all eligibility requirements and 13 were eligible for meta-analysis.

\section{F. Outcomes}

Most studies reported multiple outcomes and relationships. Across studies, the effects of similar or different personalities were frequently not the sole focus of the paper. Here we examined only outcomes associated with similar or dissimilar personalities. Of these, there were a total of 28 total outcomes. The most common type of outcome reported was robot acceptance $(86 \%)$.

For the purpose of this study the extended version of the UTAUT model proposed and validated by [31], [2] provided 
a useful framework by which to define and categorize different subgroups of acceptance. This model defines 13 subgroups of acceptance. These are anxiety, attitude, facilitating conditions, intention to use, perceived enjoyment, perceived ease of use, perceived sociability, perceived usefulness, social influence, social presence, trust, and use/usage [31, pp.529].

Utilizing this approach to categorizing acceptance outcomes, we were able to identify 24 outcomes across 17 studies that were suitable to this kind of categorization. The most common sub-component of acceptance found between these 24 different outcomes was that of intention to use (TTU) followed closely by attitude (ATT) and perceived usefulness (PU). Details can be seen in table II as to the overall counts, categories, and specific outcomes. Notably, no studies examined the acceptance constructs of: anxiety, facilitating conditions, perceived adaptability, perceived sociability, social influence, or use/usage. This void presents a potential opportunity for future researchers to examine these acceptance constructs in light of similar/dissimilar personality and its impact on acceptance of robots.

\section{RESULTS}

\section{A. Main Effects}

Table $\mathrm{II}$ and Figure 1 provide the meta-analytic results for the relationship between matching personality and acceptance of robots. The estimated true score relationship showed an overall significant and positive relationship between matching personality traits and acceptance $(k=$ $\left.13, \bar{r}^{2}=0.24,95 \% C I ;[0.14,0.43]\right)$. Based on these results, it appears that matching personality is positively related to acceptance which supports the idea of similarity attraction in the existing literature.

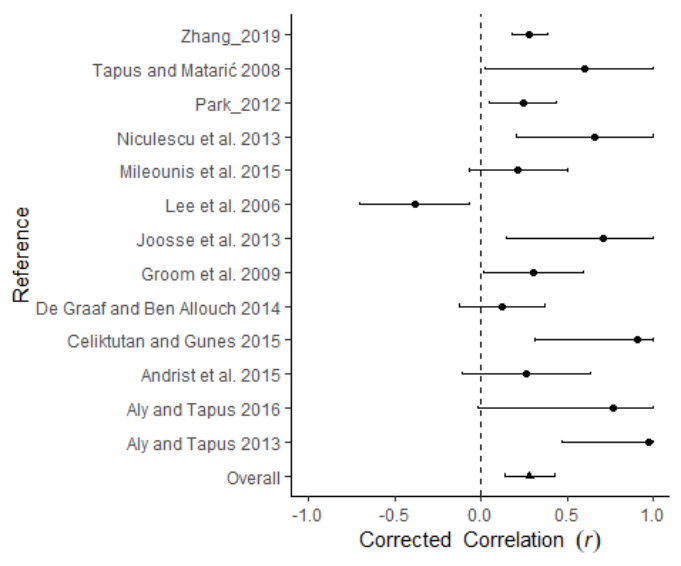

Fig. 1. Forest plot showing effect sizes for matching personalities and acceptance of robots.

Investigations of heterogeneity, however, provide a more complex picture of this relationship. Utilizing the results of $Q$ and $I^{2}$ statistics, we sought to determine the presence of potential moderators in this relationship. Results provided strong evidence of moderators impacting this relationship $\left(Q=42.1, d f=12, p<0.001-I^{2}=71.4\right)$.

\section{B. Moderators}

Across the studies examined by this meta-analysis, only a select few moderators were discernible given the relatively small number of studies ( $K$ observed) at this time. Examinations of what data do exist, however, provided support for age and gender diversity as moderators.

First, as visible in table III and figure 2, we see that personality similarity's relationship with acceptance appears to be moderated by age. The relationship is, however, only significant in the 45-64 age group and not the 18-43 age group. Within the 45-64 age group, the effect is significant and positive $\left(k=2, \bar{r}^{2}=0.24,95 \% C I ;[0.16,0.44]\right)$. In summary, the relationship between similarity and acceptance is only supported for older adults (45-64 years old).

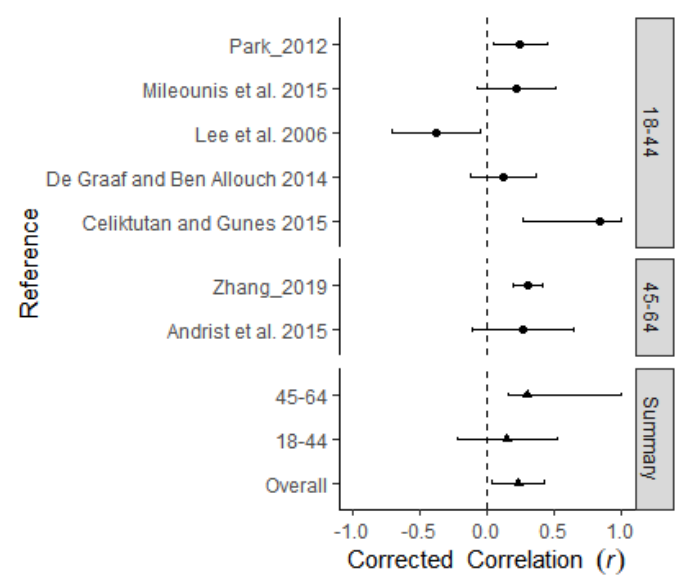

Fig. 2. Forest plot showing effect sizes for matching personalities and acceptance of robots by age group.

Second, as visible in table IV and figure 3 , we see a significant difference between two levels of gender diversity in terms of robot acceptance. Gender diversity in this study is the distribution of women to men or men to women in a study's sample. High gender diversity represents an even distribution between genders $(50 \% \pm 10 \%)$, medium diversity indicates a small skew between genders ([40\%..30\%] or $[60 \% . .70 \%])$, and low gender diversity represents a strongly skewed distribution toward one gender or another $(>30 \%$ or $<70 \%$ ). Results only support the relationship between similarity and acceptance for samples with high and medium gender diversity. However, a lack of studies with low gender diversity makes it difficult to draw conclusions about low diversity samples.

Closer examination of medium and high gender diversity individually, however, identified a statistically significant difference between high and medium gender diversity samples $\left(F=6.5,95 \% C I ;[-0.58,-0.02], r_{d}^{2}=-0.302\right)$. These results indicated a positive correlation between high and medium gender diversity and robot acceptance, with the medium gender diversity showing a significantly higher correlation than the high gender diversity group. These results provide support for the idea that samples with less gender diversity may lead to higher robot acceptance than those 


\begin{tabular}{lll}
\hline Study & UTAUT Construct & Specific Outcome \\
\hline Aly and Tapus 2013 & ATT & Perception of Personality Similarity \\
De Graaf and Ben Allouch 2014 & ATT & Perception of Personality Similarity \\
Groom et al. 2009 & ATT & Self-extension \\
Lee et al. 2006 & ATT & Social Attraction \\
Mileounis et al. 2015 & ATT & Likability \\
Park_2012 & ATT & Friendliness \\
Celiktutan and Gunes 2015 & PENJ & Enjoyment \\
Lee et al. 2006 & PENJ & Enjoyment \\
Niculescu et al. 2013 & PENJ & Empathetic \\
Niculescu et al. 2013 & PEOU & Ease of Use \\
Andrist et al. 2015 & PU & Perceived Robot Performance \\
Andrist et al. 2015 & PU & Total puzzles solved \\
Lee et al. 2006 & PU & Intelligence \\
Windhouwer 2012 & PU & Intelligence \\
Park_2012 & SP & Social Presence \\
Andrist et al. 2015 & TRUST & Total Participation Time \\
Joosse et al. 2013 & TRUST & Trust \\
Zhang_2019 & TRUST & Safety \\
Aly and Tapus 2016 & TTU & Preference for Similar Robot \\
Cruz-Maya and Tapus, 2017 & TTU & Interaction Preference \\
Dang and Tapus 2015 & TTU & Preference for Similar Robot \\
Park_2012 & TTU & Preference for Robot \\
So et al. 2008 & TTU & Preference for Similar Robot \\
Tapus and Matarić 2008 & TTU & Preference for Similar Robot \\
\hline & &
\end{tabular}

TABLE I

AtT:Attitude, Penj:Perceived enjoyment, PeOU:Perceived ease of use, PU: Perceived usefulness, SP: Social Presence, TRUSt: TRUST, TTU: INTENTION TO USE

\begin{tabular}{lllllllll}
\hline Analysis Type & Predictor & Outcome & sig & $k$ & $N$ & mean $r$ & CI_LL_95 & CI_UL_95 \\
\hline Overall & Matching Personality & Acceptance & $\mathbf{Y}$ & 13 & 980 & 0.24 & 0.1365 & 0.4280 \\
\hline
\end{tabular}

TABLE II

META-ANALYTIC RESUlts SHOWS THE ESTIMATED TRUE EFFECT SIZE BETWEEN SIMILAR PERSONALITY AND ACCEPTANCE OF ROBOTS.

\begin{tabular}{lllllllll}
\hline Analysis Type & predictor:facet & Age Range & Sig & $k$ & $N$ & mean $r$ & CI_LL_95 & CI_UL_95 \\
\hline Overall & Matching Personality & All Levels & Y & 7 & 825 & 0.2 & 0.0393 & 0.4269 \\
Simple Moderator & Matching Personality & $18-44$ & $\mathrm{~N}$ & 5 & 342 & 0.13 & -0.2244 & 0.52 \\
Simple Moderator & Matching Personality & $45-64$ & $\mathrm{Y}$ & 2 & 483 & 0.24 & 0.1603 & 0.4413 \\
\hline
\end{tabular}

TABLE III

META-ANALYTIC RESULTS SHOWING THE ESTIMATED TRUE EFFECT SIZE BETWEEN SIMILAR PERSONALITY AND ACCEPTANCE OF ROBOTS WITH AGE GROUPS AS MODERATORS.

with more gender diversity. Ultimately, a full examination of this effect was not possible here because of the lack of studies examining samples with low gender diversity, but the significant difference observed between high and medium gender diversity provides support for examining this relationship.

\section{DisCUSSION \& OPPORTUNITIES}

The goal of the meta-analysis was to examine the impact of personality similarity/dissimilarity between humans and robots on robot acceptance. The results of this meta-analysis contribute to the existing HRI literature by helping to answer this question while also highlighting where additional research is needed. To that end, the paper's findings can be organized into three overarching themes.

First, personality similarity between humans and robots does promote robot acceptance. For practitioners these results seem to support the invest needed to design robots with personalities that match their human partner 's personality. For researchers, there is also an opportunity to determine which set of similarities are more or less important.

Second, we found evidence of at least one contextual moderator in the case of age group. Age group 45 to 64 was significant while the age group 18 to 44 was not. This indicates that similarity seems to be more important to the older age group. This study demonstrates the importance of sample age in promoting robot acceptance through personality matching. Yet, these results do little to help us understand exactly why. For researchers, there is now a need to understand why we see such age differences. For robot designers targeting older adults, these results clearly show the value of matching.

Third, personality similarity was significant for both high 


\begin{tabular}{lllllllll}
\hline Analysis Type & predictor & Gender Diversity & sig & $k$ & $N$ & mean $r$ & CI_LL_95 & CI_UL_95 \\
\hline Overall & Matching Personality & All Levels & Y & 9 & 451 & 0.26 & 0.1424 & 0.5116 \\
Simple Moderator & Matching Personality & High & Y & 4 & 332 & 0.19 & 0.0995 & 0.358 \\
Simple Moderator & Matching Personality & Medium & Y & 5 & 119 & 0.49 & 0.2115 & 0.9894 \\
\hline
\end{tabular}

TABLE IV

META-ANALYTIC RESULTS SHOWING THE ESTIMATED TRUE EFFECT SIZE BETWEEN SIMILAR PERSONALITY AND ACCEPTANCE OF ROBOTS WITH GENDER DIVERSITY LEVELS AS MODERATORS.

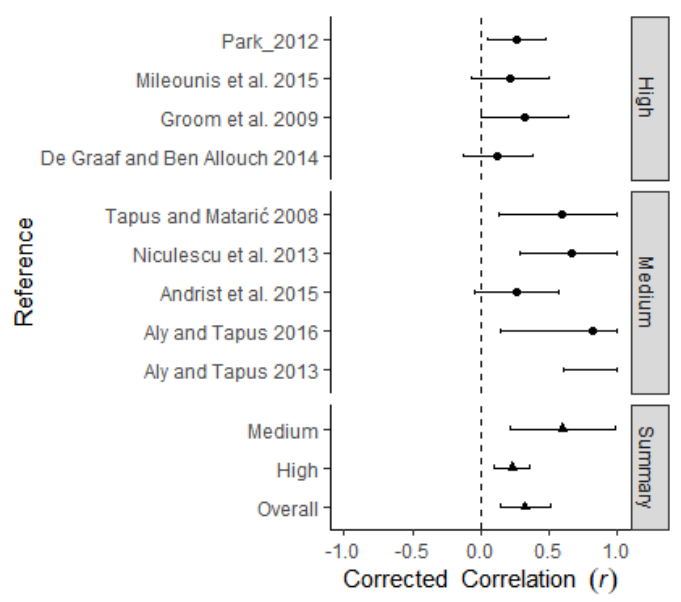

Fig. 3. Forest plot showing effect sizes for matching personalities and acceptance of robots by gender diversity.

and medium gender diversity. This effect was significantly stronger, however, for medium gender diverse samples. This finding may be useful for researchers as it suggests that potential gender differences may exist. This research opportunity is elaborated on in section V-A.1

The meta-analysis also helped to identify future research opportunities. The heterogeneity found in our overall analysis implies the presence of additional moderators. It is possible, and indeed probable, that other variables such as task type, gender, and/or culture may function as moderators in addition to gender diversity and age group. At present, however, these factors are under reported in the literature making investigation via meta-analysis ineffective and inadvisable. Thus, we present the following research opportunities.

\section{A. RO-1: Expanding Study Samples}

1) Differences Between Genders: Differences have been observed between men and women in terms of acceptance broadly [32] but also in relation to interactions with robots [33], [34]. Given the literature's focus on evenly distributed sample sizes, it is not possible to discern such differences at this time via meta-analysis. Thus future studies may wish to examine these potential differences.

2) Exploring Other Countries: Across studies collected in this review, no studies explicitly examined samples from South or Central America, North Africa or the Middle East, or Sub-Saharan Africa. This is an important gap because different national and cultural values have been found to impact acceptance of robots [35], [36].

\section{B. RO-2: Different Types of Tasks}

Social context and, more specifically, type of task have been shown to influence different outcomes related to HRI [37], [38], including the personality similarities [27]. It is entirely likely that additional social contextual factors are also relevant. However, without additional studies focused on social context and task types it is impossible to fully begin to examine this relationship. These additional studies must report data which can be used for a meta-analysis. Currently, we found only one study [27] reported sufficient data needed for a meta-analysis.

\section{RO-3: Beyond the Extroversion/Introversion Trait}

Different personality traits have different impacts across a variety of outcomes in relation to HRI [14], [5], [25]. The current literature heavily focuses on the specific trait of extroversion/introversion to the exclusion of even other Big Five traits. An exception does exist within this literature in the form of the study conducted by [39]. Further research is required to understand whether matching on specific traits has unique impacts on robot acceptance [39].

\section{RO-4: Group Level Analysis}

Although two studies in this review examined group level interactions [40], [26], there is a need for more research in this regard. Humans and robots are certain to have oneon-one interactions, but these are not the only kind of interactions that will occur [41], [1], [30], [41]. For example, most retail labor is carried out by a team or group of workers rather than one individual in isolation. Therefore, a grouplevel analysis might assist in the investigation of teams and teaming between humans and robots.

\section{CONCLUSION}

This study conducted a meta-analysis to assess the impact of personality matching on robot acceptance. Results show that matching human and robot personalities is positively related to robot acceptance. In other words, birds of a feather do indeed flock together as do humans and robots.

\section{REFERENCES}

[1] S. You and L. Robert, "Emotional attachment, performance, and viability in teams collaborating with embodied physical action (epa) robots," Journal of the Association for Information Systems, vol. 19, no. 5, pp. 377-407, 2017.

[2] C. Esterwood, K. Essenmacher, H. Yang, F. Zeng, and L. Robert, "A meta-analysis of human personality and robot acceptance in humanrobot interaction," in 39th ACM Conference on Human Factors in Computing Systems (CHI). ACM, 2021. 
[3] C. Esterwood and L. Robert, "Robots and covid-19: Re-imagining human-robot collaborative work in terms of reducing risks to essential workers," ROBONOMICS: The Journal of the Automated Economy, vol. 1, pp. 9-9, 2021.

[4] S. Shead, "Amazon now has 45,000 robots in its warehouses," Mar 2021. [Online]. Available: https://www.businessinsider.com/ amazons-robot-army-has-grown-by-50-2017-1

[5] C. Esterwood and L. P. Robert, "Personality in healthcare human robot interaction (h-hri) a literature review and brief critique," in Proceedings of the 8th International Conference on Human-Agent Interaction, 2020, pp. 87-95.

[6] S. You and L. P. Robert Jr, "Human-robot similarity and willingness to work with a robotic co-worker," in Proceedings of the 2018 ACM/IEEE International Conference on Human-Robot Interaction, 2018, pp. 251260.

[7] A. Rossi, K. Dautenhahn, K. L. Koay, and M. L. Walters, "How social robots influence people's trust in critical situations," in 2020 29th IEEE International Conference on Robot and Human Interactive Communication (RO-MAN). IEEE, 2020, pp. 1020-1025.

[8] S. You and L. Robert, "Trusting robots in teams: Examining the impacts of trusting robots on team performance and satisfaction," in Proceedings of the 52th Hawaii International Conference on System Sciences, Jan, 2019, pp. 8-11.

[9] D. Byrne and W. Griffitt, "Similarity and awareness of similarity of personality characteristics as determinants of attraction." Journal of Experimental Research in Personality, 1969.

[10] A. Tapus, C. Ţăpuş, and M. J. Matarić, "User-robot personality matching and assistive robot behavior adaptation for post-stroke rehabilitation therapy," Intelligent Service Robotics, vol. 1, no. 2, p. 169 2008.

[11] A. Tapus et al., "Stress game: The role of motivational robotic assistance in reducing user's task stress," International Journal of Social Robotics, vol. 7, no. 2, pp. 227-240, 2015.

[12] K. M. Lee, W. Peng, S.-A. Jin, and C. Yan, "Can robots manifest personality?: An empirical test of personality recognition, social responses, and social presence in human-robot interaction," Journal of communication, vol. 56, no. 4, pp. 754-772, 2006.

[13] A. Weiss and C. Bartneck, "Meta analysis of the usage of the godspeed questionnaire series," in 2015 24th IEEE International Symposium on Robot and Human Interactive Communication (RO-MAN). IEEE, 2015, pp. 381-388.

[14] L. Robert, "Personality in the human robot interaction literature: A review and brief critique," in Proceedings of the 24th Americas Conference on Information Systems, Aug, 2018, pp. 16-18.

[15] K. Abe, Y. Hamada, T. Nagai, M. Shiomi, and T. Omori, "Estimation of child personality for child-robot interaction," in 2017 26th IEEE International Symposium on Robot and Human Interactive Communication (RO-MAN). IEEE, 2017, pp. 910-915.

[16] J. Bernotat and F. Eyssel, "A robot at home-how affect, technology commitment, and personality traits influence user experience in an intelligent robotics apartment," in 2017 26th IEEE International Symposium on Robot and Human Interactive Communication (RO-MAN). IEEE, 2017, pp. 641-646.

[17] A. Cruz-Maya and A. Tapus, "Learning users' and personality-gender preferences in close human-robot interaction," in 2017 26th IEEE International Symposium on Robot and Human Interactive Communication (RO-MAN). IEEE, 2017, pp. 791-798.

[18] P. T. Costa Jr, R. R. McCrae, and D. A. Dye, "Facet scales for agreeableness and conscientiousness: A revision of the neo personality inventory," Personality and individual Differences, vol. 12, no. 9, pp. 887-898, 1991.

[19] S. Devaraj, R. F. Easley, and J. M. Crant, "Research note-how does personality matter? relating the five-factor model to technology acceptance and use," Information Systems Research, vol. 19, no. 1, pp. 93-105, 2008

[20] W. G. Graziano and N. Eisenberg, "Agreeableness: A dimension of personality," in Handbook of Personality Psychology. Elsevier, 1997, pp. 795-824.

[21] S. Speranza, C. T. Recchiuto, B. Bruno, and A. Sgorbissa, "A model for the representation of the extraversion-introversion personality traits in the communication style of a social robot," in 202029 th IEEE International Conference on Robot and Human Interactive Communication (RO-MAN). IEEE, 2020, pp. 75-81.

[22] A.-M. Velentza, D. Heinke, and J. Wyatt, "Human interaction and improving knowledge through collaborative tour guide robots," in 2019 28th IEEE International Conference on Robot and Human Interactive Communication (RO-MAN). IEEE, 2019, pp. 1-7.

[23] G. Matthews, P. A. Hancock, J. Lin, A. R. Panganiban, L. E. Reinerman-Jones, J. L. Szalma, and R. W. Wohleber, "Evolution and revolution: Personality research for the coming world of robots, artificial intelligence, and autonomous systems," Personality and individual differences, vol. 169, p. 109969, 2021.

[24] S. Rossi, D. Conti, F. Garramone, G. Santangelo, M. Staffa, S. Varrasi, and A. Di Nuovo, "The role of personality factors and empathy in the acceptance and performance of a social robot for psychometric evaluations," Robotics, vol. 9, no. 2, p. 39, 2020.

[25] L. P. Robert, R. Alahmad, C. Esterwood, S. Kim, S. You, and Q. Zhang, "A review of personality in human-robot interactions," Foundations and Trends ${ }^{\circledR}$ in Information Systems, vol. 4, no. 2, pp. 107-212, 2020

[26] O. Celiktutan and H. Gunes, "Computational analysis of human-robot interactions through first-person vision: Personality and interaction experience," in 2015 24th IEEE International Symposium on Robot and Human Interactive Communication (RO-MAN). IEEE, 2015, pp. 815-820.

[27] M. Joosse, M. Lohse, J. G. Perez, and V. Evers, "What you do is who you are: The role of task context in perceived social robot personality," in 2013 IEEE International Conference on Robotics and Automation. IEEE, 2013, pp. 2134-2139.

[28] A. W. Harzing, "Publish or perish app," 2007. [Online]. Available: https://harzing.com/resources/publish-or-perish

[29] M. J. Westgate, "revtools: An r package to support article screening for evidence synthesis," Research synthesis methods, 2019.

[30] S. You and L. Robert, "Teaming up with robots: An imoi (inputsmediators-outputs-inputs) framework of human-robot teamwork," International Journal of Research in Engineering (IJRE), vol. 2, no. 3 , 2018.

[31] M. Heerink, B. Krose, V. Evers, and B. Wielinga, "Measuring acceptance of an assistive social robot: a suggested toolkit," in $R O-M A N$ 2009-The 18th IEEE International Symposium on Robot and Human Interactive Communication. IEEE, 2009, pp. 528-533.

[32] V. Venkatesh and M. G. Morris, "Why don't men ever stop to ask for directions? gender, social influence, and their role in technology acceptance and usage behavior," MIS Quarterly, pp. 115-139, 2000.

[33] M. Heerink, "Exploring the influence of age, gender, education and computer experience on robot acceptance by older adults," in 2011 6th ACM/IEEE International Conference on Human-Robot Interaction (HRI). IEEE, 2011, pp. 147-148.

[34] P. Schermerhorn, M. Scheutz, and C. R. Crowell, "Robot social presence and gender: Do females view robots differently than males?" in Proceedings of the 3rd ACM/IEEE international conference on Human robot interaction, 2008, pp. 263-270.

[35] T. Nomura, "Cultural differences in social acceptance of robots," in 2017 26th IEEE International Symposium on Robot and Human Interactive Communication (RO-MAN). IEEE, 2017, pp. 534-538.

[36] D. Conti, A. Cattani, S. Di Nuovo, and A. Di Nuovo, "A crosscultural study of acceptance and use of robotics by future psychology practitioners," in 2015 24th IEEE international symposium on robot and human interactive communication (RO-MAN). IEEE, 2015, pp. 555-560.

[37] M. Salem, G. Lakatos, F. Amirabdollahian, and K. Dautenhahn, "Would you trust a (faulty) robot? effects of error, task type and personality on human-robot cooperation and trust," in 2015 10th ACM/IEEE International Conference on Human-Robot Interaction (HRI). IEEE, 2015, pp. 1-8.

[38] G. Canal, G. Alenya, and C. Torras, "A taxonomy of preferences for physically assistive robots," in 2017 26th IEEE International Symposium on Robot and Human Interactive Communication (RO$M A N)$. IEEE, 2017, pp. 292-297.

[39] Q. Zhang, C. Esterwood, J. Yang, and L. Robert, "An automated vehicle (av) like me? the impact of personality similarities and differences between humans and avs," in AAAI Symposium Series Technical Reports (August 31, 2019), 2019.

[40] H. Salam, O. Celiktutan, I. Hupont, H. Gunes, and M. Chetouani, "Fully automatic analysis of engagement and its relationship to personality in human-robot interactions," IEEE Access, vol. 5, pp. 705 721, 2016.

[41] S. Sebo, B. Stoll, B. Scassellati, and M. F. Jung, "Robots in groups and teams: a literature review," Proceedings of the ACM on HumanComputer Interaction, vol. 4, no. CSCW2, pp. 1-36, 2020. 